\title{
LA ENSEÑANZA \\ de la economía para el desarrollo
}

\section{ESUMEN}

En este artículo se hace un análisis sobre la enseñanza de la economía. Se describe la influencia de la academia americana en la formación de los economistas y la consolidación de la teoría neoclásica como paradigma dominante en nuestras facultades de economía. Esta es una tendencia que ha dificultado la enseñanza de corrientes científicas diferentes $y$ que ha llevado a una excesiva formalización matemática. Se analizan, además, algunos elementos que contribuyen al debate acerca de la metodología de enseñanza de la economía. Colombia requiere la formación de científicos sociales que puedan coadyuvar en la consecución de objetivos de competitividad tan necesarios para el desarrollo económico. Por ello a continuación se proponen alternativas con las que se espera mejorar los vínculos de la academia con el entorno empresarial y la actividad productiva.

\section{alabras clave}

Teoría del conocimiento en economía, economía de empresa, corriente principal, teoría neoclásica.
Por:

Gustavo Olmos Leguizamón ${ }^{I}$

\begin{abstract}
A
partir de las formulaciones keynesianas y especialmente de los trabajos de Hansen y Hicks, la ciencia económica ha sido objeto de profundos cambios en sus desarrollos temáticos, sobre todo en lo concerniente a la presentación teórica de modelos en las áreas de microeconomía, macroeconomía, teoría del crecimiento, econometría y política monetaria, entre otras.
\end{abstract}

A lo anterior se ha sumado el hecho de que autores como Samuelson y Friedman introdujeron en sus textos desarrollos pedagógicos que facilitaron la enseñanza y el aprendizaje de la economía y han proporcionado una estructura analítica esencial en sus cursos que se ha venido generalizando en los textos de las universidades occidentales de los países desarrollados y que a partir de finales del siglo XX se han popularizado en América Latina y particularmente en Colombia.

\footnotetext{
${ }^{1}$ Gustavo Olmos Leguizamón. Profesor Asistente. Economía.
} 
Este fenómeno ha sido evidente desde finales de la Segunda Guerra Mundial, cuando la enseñanza de la economía sufre un viraje sustancial. Según Misas $(2004)^{1}$, en este momento «se da un proceso relativamente rápido de internacionalización de la disciplina que toma la forma de un proceso de universalización de las prácticas y de las percepciones de la academia americana».

Los problemas de la postguerra debían ser enfrentados especialmente por los economistas de la potencia vencedora ${ }^{2}$, lo que dio lugar a una reformulación de los pénsums y de las metodologías de enseñanza. Los cambios rápidamente se generalizaron en las escuelas de economía de Europa y más tarde del resto del mundo.

\footnotetext{
1 La obra de Misas gira en torno a la epistemología económica y a la conformación del campo de la economía.

2 Correspondió a Estados Unidos, debido a su protagonismo durante la guerra y a su papel en el financiamiento de la reconstrucción europea, darle dirección a las herramientas analíticas y a las acciones de política económica para conseguir superar rápidamente los efectos del conflicto.
}

\section{A Bstract}

This article presents an analysis on the way economics is taught. It high lights - the influence American academy has had over the education of economists and the consolidation of Neo-classical theory as the dominant paradigm determining the issues and topics taught by our Economics Faculties. These factors have made it difficult to convey scientific trends that differ from the main current, making excessive mathematical formalization the way in which Economics is taught in Colombia.

Additionally, some contributing elements are presented over the debate on how economics should be taught in a country like ours, which requires the formation of social scientists able to cooperate towards the accomplishment of goals leading to the development of competitiveness, indispensable for economic growth. Also, some alternatives are proposed intended to improve the way we (the academic community) relate to the entrepreneurial environment and productive activities.

\section{KEY wORDs}

Economics theory of the knowledge, Company economics, Main current, Neoclassical theory. 


\section{LA CONSOLIDACIÓN DE LA ESCUELA NEOCLÁSICA}

La noción de teoría neoclásica abarca un conjunto muy amplio de diferentes corrientes de pensamiento. Así, por ejemplo, como neoclásicos podemos incluir a Kenneth Arrow, Milton Friedman, Gary Becker o Paul Krugman, quienes presentan entre sí grandes diferencias conceptuales.

No obstante su heterogeneidad, se impuso como modelo dominante en el aprendizaje de la economía, la enseñanza de los principios neoclásicos. La tradición clásica fue sustituida por la necesidad de imponer los fundamentos de la teoría neoclásica, convertida por la academia americana en la ciencia económica.

La puesta en práctica de este modelo en los países subdesarrollados ha llevado a un divorcio creciente entre la teoría, las herramientas y el mundo real de la economía, porque como resultado hemos desembocado en una concepción funcional de la economía, que si bien nos hace hábiles en el manejo de los instrumentos, especialmente de tipo matemático, nos incapacita para pensar la economía de nuestro país. Para entender nuestro entorno económico debemos añadir una gran dosis de creatividad y hacer un gran esfuerzo para problematizar lo aprendido o para darle sentido a los saberes adquiridos mediante la respuesta a los interrogantes que surgen de nuestra realidad.

Como afirma Bejarano (1997) la enseñanza de la economía es un proceso de transmisión del conocimiento económico que adopta la forma de programas académicos y curriculares de nivel universitario, que al ser mediada por el uso de textos simplificadores originados especialmente en Estados Unidos, no consulta nuestra realidad. Este hecho acentúa los obstáculos epistemológicos de la enseñanza de la economía, puesto que se quiere llevar al estudiante a pensar sobre los problemas económicos reales, por encima de sus preconceptos intuitivos o de sus ideas partiendo de un entorno ajeno al que le corresponde en su sociedad.

El hecho de que en la enseñanza de la economía se reserve un lugar central a la tesis neoclásica obliga a que en nuestros programas se incluya un abultado número de cátedras que se ajusten a sus fundamentos conceptuales, lo cual contribuye a homogeneizar los contenidos de los programas académicos para las distintas facultades de economía de nuestro país, mediante la puesta en marcha de las propuestas doctrinarias de la citada escuela.

El creciente divorcio entre los hechos económicos (el mundo real de la economía) y la abstracción que de ellos hace la teoría neoclásica ha sido uno de los motivos por los cuales en muchos países se está replanteando lo que se enseña en nuestra carrera ${ }^{4}$, circunstancia propicia para originar nuevas propuestas que alimenten el debate sobre la formación de los economistas que requiere un país en desarrollo como Colombia.

A lo anterior agrega Currie (1967): «El mayor valor de una buena educación económica radica en la forma de pensar y en los métodos de abordar los problemas que ella confiere». Para alcanzar este objetivo, sin embargo, se requiere no solamente un cambio en la manera de enseñar, sino también una disminución en el énfasis puesto sobre la formalización que se enseña a partir de la corriente dominante (la neoclásica). Si alguna pertinencia tiene la formación que se imparte al economista, esta 
consiste en su orientación hacia la promoción y el fomento del espíritu investigativo y la creatividad. El objetivo debe ser contar con profesionales capaces de pensar con cabeza propia y sin grilletes ideológicos. Aunque los conocimientos sean importantes, es fundamental trabajar sobre la capacidad de pensar y sobre la idoneidad para el discernimiento.

\section{Tendencias en la enseñanza de la economía}

De acuerdo con indagaciones preliminares realizadas en la EAN ${ }^{1}$, referentes a tendencias en la educación superior para facultades y programas de economía, se han podido establecer algunas tendencias y rasgos comunes, especialmente en aspectos tales como: planes de estudio, contenidos y formación investigativa. Sobresalen las siguientes características:

- Tendencia a reducir la duración de programas de Economía a cuatro años.

- Mayor relevancia de materias del área de teoría económica, tales como microeconomía y macroeconomía.

- Menor importancia a asignaturas del área de economía política.

- Incremento en enfoques instrumentales en materias como estadística y econometría.

- Inclusión del idioma inglés como exigencia curricular específica o como exigencia de conocimientos al ingreso a cursar estudios de economía.

- Una notable disminución de la investigación en la formación curricular de pregrado, atribuible al creciente desarrollo de los postgrados, nivel éste donde se privilegian las actividades investigativas.

\section{Comprensión y uso del instrumental matemático}

La herramienta matemática es considerada de gran utilidad práctica para el diseño de soluciones a los problemas de la sociedad a fin de transformar fenómenos en beneficio de la comunidad. De igual manera contribuye con los procesos de investigación económica y social, siempre y cuando sea usada como instrumento y no como fin en sí mismo.

La discusión sobre este tópico se ha originado en el hecho que los estudios económicos se deben impartir al estudiantado con desarrollos analíticos que en su gran mayoría se fundamentan en la formalización de modelos matemáticos.

El Decreto No. 940 de 10 de mayo de 2002 del Ministerio de Educación Nacional y normas complementarias, que estableció los estándares mínimos de calidad en programas profesionales de pregrado en economía, dispuso en su artículo IV que «el manejo de los principios económicos exige la comprensión de métodos analíticos y argumentos basados en modelos». Esta reglamentación condiciona al profesorado a aplicar una metodología que responda al requerimiento de Ley, conducente al logro de los estándares de calidad en la enseñanza de la economía, exigidos por las entidades competentes en el orden académico.

Sobre este particular, podemos citar a economistas de países hispanos tan reconocidos como Argandeña, Gámez y Mochón (1996), que se han referido a la economía como «una ciencia que opera con modelos (es decir, que aprendemos economía

\footnotetext{
${ }^{1}$ Se anota el caso del debate sobre la enseñanza de la economía en Francia, publicado en el número 32 de la Revista Cuadernos de Economía de la Facultad de Ciencias Económicas de la Universidad Nacional de Colombia.
} 
mediante modelos), y que, por ello, es necesario conocer bien las potencialidades $y$, sobre todo, las limitaciones de los distintos modelos». Esta visión no desconoce el carácter social de la economía como ciencia del hombre, pero considera que el uso del leguaje matemático y de los modelos optimiza los recursos del aprendizaje y consigue más eficazmente que los estudiantes lleguen a pensar como economistas.

Así las cosas, todo apunta a que la fundamentación matemática a nivel de pregrado se debe fortalecer como herramienta de análisis y comprensión de la teoría económica, especialmente cuando se trata de preparar a los estudiantes para que cumplan con los estándares académicos internacionales en matemáticas con el objeto de incentivar la formación en economía a nivel de maestrías y doctorados y facilitar la admisión en las mejores universidades del mundo.

En síntesis, lo que se sugiere es fortalecer la formación matemática, sin descuidar la formación en la aplicación de las herramientas para comprender los problemas económicos reales, priorizando las acciones destinadas a mejorar la creatividad, la capacidad de análisis y la comunicación.

Mc Closkey (1985) plantea que la economía puede aprender mucho de la lingüística y de la crítica literaria como disciplinas, por su habilidad para desentrañar las formas y los contenidos de la literatura. Según este autor norteamericano, los economistas sustentan sus prejuicios por medio de ecuaciones complejas y pruebas econométricas, es decir, que la economía ha adquirido un lenguaje más matemático formal que literario.

Para Mc Closkey, la retórica no es una práctica peyorativa, no remite a un discurso hueco, sino por el contrario a la forma cómo se pueden argumentar y explicar los fenómenos ocurridos en las ciencias, las profesiones y la literatura. En otras palabras, la claridad y la argumentación literaria podrían mejorar la comunicación entre los economistas y se incrementaría el número de personas que podrían comprender los principios de la ciencia económica y los fenómenos que involucra, más allá de las matemáticas.

Desde esta perspectiva resulta igualmente importante dar prioridad al aprendizaje del inglés, pues en esta lengua se accede con mayor facilidad y prontitud a los nuevos desarrollos, hallazgos y contribuciones de la ciencia económica. En esta lengua se comunican, entran en contacto y dan a conocer sus aplicaciones la mayoría de los investigadores, sin depender de las traducciones. El dominio de una segunda lengua enseña a pensar, gracias al dominio de una estructura diferente a la utilizada diariamente en lengua nativa.

\section{La formación integral}

El economista debe ser un profesional culto y dispuesto a aprehender los cambios en las ciencias y los que a diario se dan en su profesión. Para ello debe incursionar con autodisciplina en la lectura compleja (ser un estudiante toda la vida), en aras de mantenerse actualizado con las nuevas corrientes de pensamiento en el ámbito de su profesión.

El economista debe integrar conocimientos (el saber), habilidades (el hacer) y valores (el ser). Para el cumplimiento de esta formación se debe tomar la investigación como instrumento de refuerzo y de orientación docente que integre al hombre y la realidad (medio); de igual manera, que le permita contactar y validar las teorías y las corrientes del pensamiento económico con el entorno de interés. 
Hay premios Nóbel que se apartan de la ortodoxia económica, como William Fogel, Buchanan, Amartya Sen y el psicólogo Daniel Kahneman que obtuvo el premio Nóbel de Economía en el 2003. Algunas de sus obras pueden servir de apoyo para la revisión total de las teorías micro y macroeconómicas, en especialmente Kahneman analiza la toma de decisiones en economía, un proceso no tan racional como supone la teoría neoclásica.

El conocimiento en temas de producción manufacturera y de servicios permite mejorar la puesta en práctica de un cambio con énfasis teórico hacia temas centrados en la realidad, como los de economía de empresa. También los conocimientos detallados sobre comercio exterior pueden ampliar los conocimientos de geografía de la carrera, con tratamientos específicos según los productos y los países de interés. También es básico el estudio detallado y profundo de la región que el profesional quiera contribuir a desarrollar tanto desde el punto de vista humano como geográfico y productivo.

\section{Hacia un currículo integral en economía}

Un currículo es más que los conocimientos que contiene, pues abarca el compromiso de los estudiantes con las ofertas que se le plantean. En cualquier caso es la presentación de lo que se considera importante como conocimiento válido. El ordenamiento y la presentación de estos elementos en un currículo reflejan una sensación por parte del educador respecto de lo que es importante como acto genuino de conocimiento.

Si se quieren actualizar las propuestas académicas de los programas de economía, es necesario plantear un currículo integral, que tenga en cuenta el papel asumido por la

\section{Si se quieren actualizar las}

propuestas académicas de los

programas de economía, es

necesario plantear un

currículo integral, que tenga

en cuenta el papel asumido

por la profesión,

profesión, que priorice temas como productividad, competitividad, riqueza, eficiencia y crecimiento económico.

Pero dado que en el mundo la enseñanza reserva un lugar central a la tesis neoclásica, esto limita la implementación de un currículo integral, porque los estudiantes son conducidos a creer no solamente que la teoría neoclásica es la única corriente científica, sino también que su cientificidad se explica por su carácter axiomático o por el uso sistemático o incluso exclusivo de la modelización formal. En tanto constituye el componente fundamental de lo que se enseña en las mejores universidades colombianas, se convierte en gran parte del contenido de los exámenes de estado (ECAES), requisito que valida las exigencias institucionales de la academia colombiana.

Complica lo anterior, el hecho de que los economistas asistamos pasivamente a una evidente carrera hacia la monopolización de las grandes empresas de producción y de servicios, sin hacer reflexión académica alguna sobre la necesidad de impulsar los diferentes sectores económicos y enfatizar en el estudio de la empresa como una forma de contribuir al desarrollo de nuevas propuestas que lleven a la solución de nuestros problemas de empleo y desarrollo. 
Por esto, la formación universitaria fundamentalmente debe proveer a los estudiantes de economía de la capacidad de análisis para identificar, abordar y proponer soluciones a los problemas reales, basada en la adquisición de conocimientos sólidos sobre la teoría y el uso de herramientas aplicadas al conocimiento del entorno económico.

A esto se deben sumar el análisis de procesos de producción manufacturera y de servicios. Según Nieto Potes (2005) «la orientación diferenciadora de los programas tradicionales que podría tener el programa de Economía de Empresas de la EAN es la de formar profesionales competentes en el análisis y asimilación del comportamiento presente y futuro de los entornos de políticas generales de largo plazo, macroeconómicas, sectoriales y de cadenas productivas para efectuar recomendaciones $y$ diseñar políticas $y$ programas que apoyen el desarrollo de la organización y le permitan adaptarse a los cambios en dichos entornos con agilidad».

No hay que olvidar que los cambios en el entorno macroeconómico en un mundo globalizado afectan el desempeño de las organizaciones y que son los economistas los llamados a señalarlo. En palabras de Nieto Potes (2005), «la existencia de déficits de balanza comercial durante períodos prolongados en competencia con la producción local puede conducir a crisis agravadas como la de 1998-1999. De hecho, esta crisis fue precedida por un déficit de balanza comercial acumulado durante casi siete años. Igualmente, variaciones de la tasa de cambio por afluencia de "capitales golondrina», un mayor o menor crecimiento de las importaciones de la región latinoamericana, una política fiscal expansiva, variaciones de la tasa de interés en los EE.UU., cambios inducidos en las estructuras de un determinado sector, conformación de asociaciones para aprovechar las ventajas de una cadena productiva y muchos otros fenómenos de los entornos meta y macro señalados, afectan cada día más claramente y con mayor fuerza a las organizaciones».

En respaldo de lo anterior, hay que anotar que el desempleo es uno de los fenómenos más estudiados por los economistas y que los estudios sobre desempleo han proporcionado importante información para la generación de políticas tendientes a disminuirlo; no obstante, al observar el escenario laboral colombiano, encontramos que según cifras del DANE, la tasa de desempleo urbano a febrero de 2006 bordeaba el $16 \%$, lo cual dice mucho de la poca efectividad de la política macro-económica.

Asimismo, las estadísticas sobre el comportamiento del mercado laboral en el periodo enero - julio 2005 indican que el 40\% del total de la población se ocupó principalmente como trabajador por cuenta propia, mientras que el $34 \%$ lo hizo como obrero y empleado particular; es decir, que el $74 \%$ del total de la población, se ocupó en labores privadas y no estatales y muy seguramente en ese porcentaje podemos encontrar a un número importante de profesionales, entre ellos economistas.

Visto lo anterior, el problema surgiría al considerar la formación que debe recibir el economista para enfrentar esta realidad. Tradicionalmente nuestras universidades han formado sus economistas con una visión y un enfoque hacendista, orientado al manejo de políticas de corte macroeconómico, principios que sólo un grupo selecto de profesionales tiene la oportunidad de aplicar, debido al exiguo número que logra vincularse a instituciones estatales. 
El otro gran grupo de economistas debe explorar las posibilidades que ofrece el sector privado, en la mayoría de los casos como empleados y solo en un porcentaje mínimo como empresarios, en áreas de trabajo donde se requieren profesionales de la economía que conozcan del enfoque mesoeconómico.

\section{Consideraciones finales}

A partir de la década de los noventa la economía colombiana está aplicando una nueva estrategia de crecimiento económico ${ }^{1}$. Esta estrategia que devuelve al mercado su papel fundamental como eje en el proceso de asignación de recursos para conseguir el desarrollo, debe tener una respuesta desde la academia, para que las acciones de política económica no queden truncas y puedan ser impulsadas por economistas profesionales que respondan a este cambio de rumbo en la dirección económica de nuestra sociedad.

La actividad económica del sector privado genera un alto porcentaje de la riqueza nacional, lo cual la convierte en un objetivo prioritario para el estudio y el análisis por parte de los estudiantes de economía dentro de su proceso de incorporación a la práctica profesional, ya sea en empresas privadas o como emprendedores que desarrollan sus propios proyectos empresariales.

Este es un reto que nos lleva a preguntarnos qué tipo de economistas necesitan las empresas, qué habilidades deben desarrollar, qué actitudes deben tener y qué conocimientos deben adquirir los estudiantes de economía para mejorar en el ejercicio profesional.

Hasta el momento, el trabajo en el sector privado colombiano ha sido un campo propicio para el ejercicio profesional de los administradores de empresas, de los ingenieros, de los abogados o de otras profesiones que se vinculan como parte de su actividad profesional. Sin embargo, los egresados de los programas de economía deben comenzar a proyectar su desarrollo profesional revisando las oportunidades que ofrece la actividad productiva del sector privado. Una de las características del desarrollo empresarial colombiano ha sido la marcada tendencia hacia la creación de negocios. Pero la visión a corto plazo en muchos de estos proyectos apenas ha permitido su supervivencia $y$, solo en algunos casos, crecimientos moderados, a diferencia de aquellas empresas que contemplan en su análisis tanto una mirada a corto, mediano y largo plazo.

No es desconocido para nadie el elevado porcentaje de organizaciones que opera en el mundo de la informalidad. A éste respecto, según cifras del DANE para el año 2000, el $51 \%$ de la población ocupada en el sector informal tenía estudios secundarios y un 13\% estudios universitarios. Entre las actividades económicas, en el año 2004 el sector comercio ocupaba un $39 \%$, seguido por el sector de servicios y la industria.

Otro aspecto que debe analizarse es que el $70 \%$ de la población oscila entre los 25 y los 55 años de edad. En términos generales, la restricción de tiempo de las personas ocupadas en el sector informal impone barreras al estudio, disminuyendo así la posibilidad de revertir la tendencia creciente del sector informal en la economía. De allí la importancia de generar estrategias pedagógicas desde las universidades que contribuyan a apoyar la ampliación de los niveles de formación de dicha población.

\footnotetext{
${ }^{1}$ Currie Lauchlin. La enseñanza de la economía en Colombia. Ediciones Tercer mundo. 1967
} 


\section{Los clusters, las cadenas de} valor y las relaciones de estas

con otras instituciones,

también conforman el

programa de la

mesoeconomía

abastecimiento (supply chain), de los procesos de innovación y crecimiento, y de aquellos procesos donde el economista pueda integrarse.

Si al conocimiento necesario para responder las preguntas mencionadas le agregamos el conocimiento que el economista tiene acerca del funcionamiento de la economía, de la inflación, de la distribución del ingreso, del empleo, de los efectos de la política económica, los economistas podrían ofrecer mejores elementos

Por lo anterior, se hace necesario orientar los planes de estudio de los economistas hacia el conocimiento de las empresas y de la mesoeconomía (sector, territorio, cadenas); es decir, la economía de empresa, entendida como la materia que se unifica temáticamente en torno al análisis de los elementos que coadyuvan a la creación de las ventajas competitivas y que incluyen los trabajos sobre competitividad de Porter, que en nuestro país se han convertido en el fundamento del impulso a las cadenas de valor $y$ a los estudios sobre regiones competitivas, sobre todo a partir de los trabajos de la firma Monitor de mediados de los 90 del siglo XX.

También, según López de Mesa (2006), «los trabajos de competitividad desde Porter adquirieron estatus de categoría analítica en las ciencias de la gestión. Hoy hacen parte de las cadenas productivas, los estudios sobre regiones competitivas, los acuerdos comerciales, la agenda interna de los países y las interrelaciones entre macroeconomía e instituciones que intervienen en el desarrollo empresarial, por ejemplo, las universidades y el sistema educativo. Los clusters, las cadenas de valor y las relaciones de estas con otras instituciones, también conforman el programa de la mesoeconomía».

Para esto habrá que tener en cuenta el estudio de los procesos de gestión y operación de las cadenas de valor y de las cadenas de para la toma de decisiones y proporcionar información que otras profesiones no pueden ofrecer por la misma naturaleza de su formación.

Siguiendo con esta reflexión, se podría pensar por un momento qué tipo de habilidades debemos desarrollar en nuestro estudiante. ¿Debe ser capaz de ser un gerente? ¿Debe ser capaz de ser un directivo? ¿Debe ser capaz de pensar estratégicamente? ¿Debe ser capaz de identificar oportunidades de negocios con crecimientos rentables y sostenidos? Si debe ser capaz de conseguir lo que acabamos de señalar, ¿qué instrumentos debe manejar? ¿Con qué tipo de información debe estar familiarizado? Sobre todo, ¿cómo podría identificar oportunidades?

Para resolver en especial esta última pregunta, se debiera desarrollar exhaustivamente lo que se refiere a la formación de emprendedores, es decir, que los economistas sean capaces de generar proyectos empresariales individuales 0 vinculados a la sociedad o a las empresas establecidas. Impulsar emprendedo-res debe estar enmarcado en un profundo conocimiento de la economía nacional pero también en un desarrollo preciso de habilidades gerenciales y ejecutivas de los estudiantes, y vinculado a programas de apoyo a las empresas.

Todos los elementos mencionados permiten vislumbrar la complejidad que puede 
representar la integración de la Facultad de Economía con la sociedad y con la economía del país. Primero es, desde luego, necesario organizar las tareas que nos lleven a cumplir estos objetivos. En la medida en que consigamos estructurar y poner en marcha este proceso, estaremos en la capacidad de fortalecer el énfasis en economía de empresa y mejorar el perfil de nuestros egresados.

En países como el nuestro, donde el sector privado no investiga, la universidad debe desempeñar un papel preponderante en el desarrollo de la investigación científica de la economía. Ella es la encargada de percibir los hechos de manera sistemática, de ubicarlos temporal y espacialmente; de analizar su naturaleza y clasificarlos; de establecer la relación entre las partes e integrar el todo a partir de las distintas ramas de la actividad económica.

En el sector privado, los economistas desempeñan un papel muy importante. Por ejemplo, en el sector financiero el $30 \%$ de los miembros de las Juntas Directivas de los bancos son economistas de profesión, que responden por la comprensión del entorno local e internacional, como parte esencial en la conducción de dicho sector. También en los gremios económicos y a nivel medio y ejecutivo se nota la presencia de economistas, razón más que suficiente para que continuemos trabajando en el direccionamiento de nuestro currículo hacia la incorporación de los aspectos mencionados.

\section{Bibliografía}

ARGANDOÑA, Antonio; GÁMEZ, Consuelo; y MOCHÓN, Francisco (1996). Macroeconomía Avanzada I. Editorial McGraw Hill.

BEJARANO, J. (1997). Evaluación del Estado de las Disciplinas Económicas en Colombia; Un Enfoque Institucional. Universidad Externado de Colombia.

COATS, A.W. (Ed) (1996). The Post- 1945 Internationalization of Economics. Supplement to volume 28 of History of Political Economy. Durham: Duke University Press.

CURRIE, Lauchlin. La enseñanza de la economía en Colombia. Ediciones Tercer mundo. 1967.
DEBREU, G. (1991). The Mathematization of Economic Theory. American Economic Review, Vol 81, Issue 1.

DOMINGO, Rosario; y TANSINI, Rubén. Relevamiento sobre educación e investigación en Economía en América Latina. Departamento de Economía Facultad de Ciencias Sociales. Universidad de la República. Montevideo (Uruguay). 1997.

FITOUSSI (2001). L'Enseignement Superior de l'Economie en Question. Anexe 1, París, Fayard.

HARBERGER, A. (1996). Good Economics Comes to Latin American 1955-1995. En: COATS, A.W. (Ed.) (1996). 
HERRERA VALENCIA, Beethoven (2005). Evaluación de la Enseñanza de la Economía en Colombia. Faltan datos editoriales

HERRERA, B. (2004). Marco de fundamentación conceptual y especificaciones del ECAES de economía. Asociación Colombiana de Facultades y Departamentos de Economía, AFADECO, ICFES. Bogotá.

KALMANOITZ, Salomón (1997). Los medios y los públicos a las puertas del siglo XXI. Revista Panorama Económico. Universidad de Cartagena. Facultad de Ciencias Económicas Revista No.6.

KOLM, S.C. (1988). Economics in Europe and in the USA. European Economic Review, 32.

LEONTIEF, W. (1982). Academic Economics. Science, vol 217.

LÓPEZ DE MESA, Jaime O. (2006). El Estado del Arte en el Estudio de la Economía de Empresa, Propuesta para adelantar el estudio de diseño de la especialización en economía de empresa. Faltan datos restantes.

MCCLOSKEY, Donald (1985). Retórica de la Economía. Alianza Editorial. Faltan datos editoriales.

MISAS, Gabriel (2004). El Campo de la Economía y la Formación de los Economistas. (faltan datos editoriales) Cuadernos de Economía, v. XXIII , n. 40, Bogotá.

MORALES, Fabio (1997). Eficiencia e intercambio.Corriente neoclásica, institucionalismo y neoinstitucionalismo. Cuadernos de economía Vol. XVI, No. 26.

NIETO POTES, Mauricio (2005). Conceptos preliminares sobre el objeto de estudio y las competencias del egresado. Documento elaborado a propósito de la evaluación de la propuesta del programa de economía de empresa en la Universidad EAN. Bogotá, Universidad EAN. Vicerrectoría de Investigaciones.

PALACIOS, M. (2001). Saber Es Poder: el Caso de los Economistas Colombianos. En: PALACIOS, Marco (2001). De populistas, mandarines y violencias, luchas por el poder. Editorial Planeta Colombiana, S.A. Bogotá.

POSNER, George (2000). Análisis de Currículo. Editorial McGraw Hill.

OSORIO, Margarita; y otros (1999). Las prácticas evaluativas. Una reflexión necesaria en el contexto de la educación superior. Revista Investigación Bolivariana. Universidad Simón Bolívar. Instituto de Investigación. Revista No. 2.

SEN, Amartya. On Sustainable Human Development.

STIGLER, George (1982). El economista como predicador y otros ensayos. Barcelona, Orbis.

CONSEJO NACIONAL PROFESIONAL DE ECONOMÍA. Documento Reflexiones Económicas sobre Colombia. IV y V Encuentros Nacionales de Decanos y Directores de Economía. 
